\title{
DEVELOPMENT OF A CAI SYSTEM OF STANDARD SOLUTIONS BASED ON TRIZ
}

\author{
Bojun Yang, Runhua Tan, Yumei Tian \\ School of Mechanical Engineering, Hebei University of Technology, TianJin, 300130, \\ China; Email: ybj@hebut.edu.cn
}

\begin{abstract}
Standard Solutions is a powerful tool based knowledge for quickly finding effective solutions to complex product design and production problems. As an independent part of TRIZ, it can be used to solve level three inventive problems in product design for innovation. Standard Solutions is usually used with Su-F Model at first step. The CAI system InventionTool was developed using an algorithm concerning Standards, different Standards were selected according various constraints. The development process of software supported by Object-Oriented method, $\mathrm{VC}++$ language and XML.
\end{abstract}

Key words: Standard Solutions; Theory of Inventive Problem Solving; Object-Oriented Method; XML; Computer Aided Innovation

\section{INTRODUCTION}

TRIZ is a useful tool and an important the instructional theory for product innovation design. As an independent part of TRIZ, 76 Standard Solutions can be used to solve relatively common optimization problems, and more particular to solve level three inventive problems ${ }^{1}$. With the help of software designer can make more effective and more doable product design scheme, and that is the aim of Computer Aided Innovation. Many CAI software based TRIZ have been developed, such as TRISolver, IWB, Goldfire and so on. But to using Standard Solutions, no software has a maturity implement. In the CAI software Invention Tool, Standard Solutions

Supported by the Project of Natural Science Foundation of China under Grant No.50375045, the Project of Natural Science Foundation of Tianjin under Grant No.043802211

Please use the following format when citing this chapter:

Yang, Bojun, Tan, Runhua, Tian, Yumei, 2006, in International Federation for Information Processing (IFIP), Volume 207, Knowledge Enterprise: Intelligent Strategies In Product Design, Manufacturing, and Management, eds. K. Wang, Kovacs G., Wozny M., Fang M., (Boston: Springer), pp. 477-482. 
had been developed a module of software through Object-Oriented method and XML software design method.

\section{STANDARD SOLUTIONS}

\section{$2.1 \quad$ TRIZ}

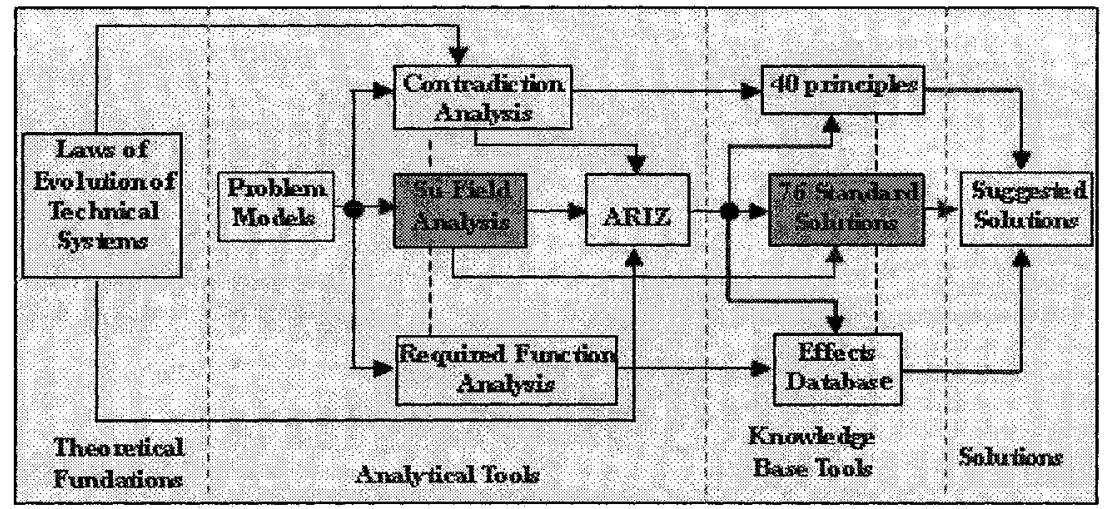

Figure 1. Structure of TRIZ Methodology

$\mathrm{TRIZ}^{2}$, the methodology of inventive problem solving, is developed by G. S. Altshuler from 1946. TRIZ specialists have taken about 3500 man-years and analyzed approximately 2.5 million patents of all over the world for the development of the theory. After more than 50 years development, TRIZ become a powerful methodology for technical problem solving that leads to enhancement of existing technique and strong acceleration of progress. TRIZ has been defined a human-oriented knowledge-based systematic methodology of inventive problem solving. According to the process of inventive problem solving, TRIZ system constructed from the theoretical foundation, the analytical tools and knowledge base tools (Figure 1). The key step of design for innovation is to produce new concepts in conceptual design stage. The knowledge is needed to support designers at that stage. Standard Solutions is a kind of TRIZ knowledge ${ }^{3}$ (Table 1).

Table 1. The knowledge to support inventive problem solving

\begin{tabular}{lll}
\hline Knowledge & Search engine & Levels of solutions \\
\hline 40 principles & 39 problem parameters and Matrix & 2 \\
76 Standard Solutions & Su-Field Model & $2,3,4$ \\
Effects & 30 TRIZ standard functions & $2,3,4$
\end{tabular}




\begin{tabular}{lll}
\hline Knowledge & Search engine & Levels of solutions \\
\hline TE patterns and paths & TE patterns & $2,3,4$ \\
\hline
\end{tabular}

\subsection{Substance-Field Model}

Substance-Field, or Su-F, is a graphical model of a minimal working technique in TRIZ ${ }^{4}$. Figure 2 is a simple description of Su-F Model. As a rule, a Su-F model comprises three components: two substances and a field usually. The identification of substances depends on the application, often $\mathrm{S} 1$ is a product or a raw object and S2 is a tool. Su-F Models and Su-F Analysis, created by G. S. Altshuller, provide a fast, simple description of subsystems and their interaction in a technical system via a well-formulated model of the technique in which all subsystems, inputs, and outputs are known or can be quite easily determined. Su-F Model is one of the most analysis tools in TRIZ, usually used in ARIZ with Standard Solutions just as Figure 1 shown. Figure 3 shows a complete description of solving a problem in Su-F model.

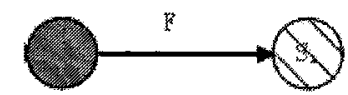

Figure 2. a simple Su-F Model

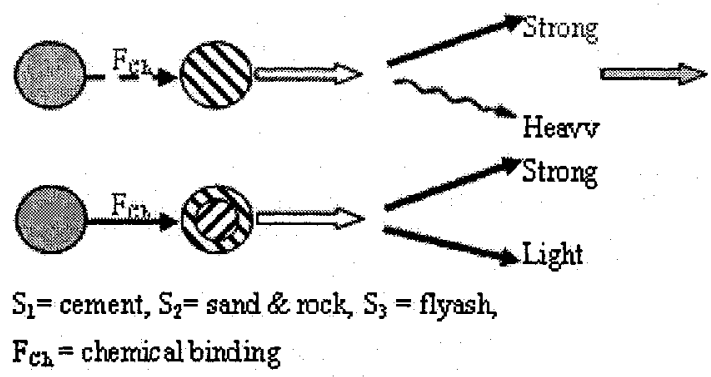

Figure 3. complete process of solving a problem in Su-F Model

\section{$2.3 \quad 76$ Standard Solutions}

Altshuller discovered various engineering problem from different fields can be solved by generic conceptual approaches. He formulated and systematized these approaches, known as TRIZ Standard Solutions or Standards. The Standards is a powerful tool based knowledge for quickly 
finding effective solutions to complex product design and production problems. They are grouped into 5 large categories as Table 2:

Table 2. The Standard Solutions categorization

\begin{tabular}{ll}
\hline 1. Improving the system with no or little change & 13 standard solutions \\
2. Improving the system by changing the system & 23 standard solutions \\
3. System transitions & 6 standard solutions \\
4. Detection and measurement & 17 standard solutions \\
5. Strategies for simplification and improvement & 17 standard solutions \\
\hline
\end{tabular}

The 76 Standard Solutions are used to solve relatively common optimization problems. Further more, they are useful for level three inventive problems. Level 3 inventions significantly improve existing systems, and represent $18 \%$ of the patents. Typically, the 76 Standard Solutions are used as a step in ARIZ, after the Su-F Model has been developed and any constraints on the solution have been identified. Standards and Su-F are linking tightly each other. Before use Standards, a Su-F Model of technical problem must be constructed mostly.

Standards are the precepts of synthesis and transformation with the aim to overcome or circumvent technical and physical contradictions. ${ }^{4}$ When a problem is solved with the help of Standards, there is no need to formulate a contradiction. For easy to orient in Standards, some algorithms for choosing the right Standards had been designed. In an algorithm which is base for development of the CAI system concerning Standards, different Standards were selected according various constraints.

\section{THE CAI BASED TRIZ}

After more than 50 years development and many successful applications in superior companies in the world, TRIZ is regarded as a useful innovation methodology in practice. Computer Aided Innovation software based on innovational theory is a kind of CAD that is suitable for conceptual design stage $^{5}$. TRIZ-based software as the main content of CAI developed rapidly in recent years.

TRISolver $2.1^{\mathrm{TM}}$ is the early English-language version of the CAI software from TRISolver Consulting Group in Germany. The Invention Machine in America had issued TechOptimizer, and than upgrade to Goldfire Innovator. Another America firm Ideation International developed the Innovation WorkBench (IWB) and so on. Those English-language software of CAI integrate the concepts, principles and tools of TRIZ. Those CAI software were applied in practice and made great success. But to use of Standards, some of them mention little or no, some is just a database or a 
guide to Standards knowledge. How to use the Standards in CAI software need develop.

In china, some kinds of English-language version software such as Goldfire Innovator were introduced. For the limitation of language and thinking custom, that kind of software is hard success completely. Many Chinese-language version CAI software were developed by university or institute organization. But most of them are experiment. InventionTool1.0 and 2.0 based on TRIZ had been completed by Hebei University of Technology and were in commercial. ${ }^{6}$

\section{THE DEVELOPMENT OF STANDARDS SYSTEM}

According to the algorithm for using 76 Standard Solutions, a Standard Solutions module of InventionTool CAI software is developed. In one CAI software it is realized not only to support technical knowledge but to solve problem using in 76 Standard Solutions systemically.

A software development process is fallen into 6 steps in term of the lifecycle of software: analysis by Fusion method, design, implementation, module test, system test and executing. The Standard Solution module follows the same process. Object-Oriented method $^{7}$ is the mainstream method to develop software. Fusion method integrated and expended the advanced characteristics of some other Object-Oriented methods. The module analysis obeys Fusion method, and implement with $\mathrm{VC}++$ language and XML.

Several object models have been created during the software development process. Each object model presents one aspect of the software. The total of object models make up of the object model of Standards System. A life-cycle model of the Standards module includes operators occurring orders, operations is then established.

XML (eXtensible Markup Language) is used to develop the interface of the software. XML Standard by W3C (World Wide Web Consortium). For its extensible, structured and self-describe data, XML is more impactful then HTML. This software use a mode of XML+XSL $\rightarrow$ HTML. On its strong suit of data transfer and data store, XML also can be used to update the CAI software.

\section{CONCLUSION}

With minutely analyzing on TRIZ method, Su-F model and 76 Standard Solutions, according to the algorithm for using Standard Solutions, the 
Standard Solutions module software is completed. The development process supported by Object-Oriented method, VC++ language and XML. Compared with others CAI software based TRIZ in the world, InventionTool and its Standard Solutions module have their own characters and value.

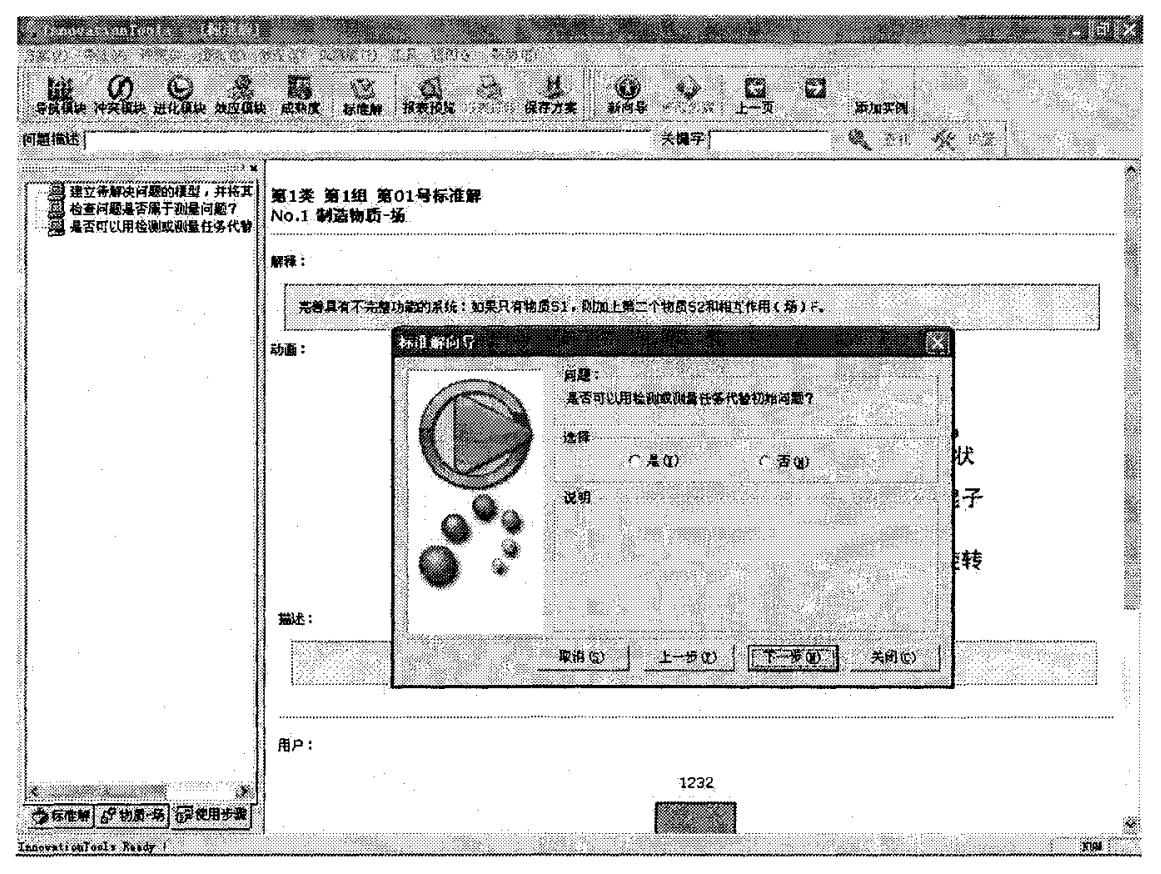

Figure 4. interface of Stanards Module

\section{REFERENCES}

1. John Terninko, Ellen Domb, Joe Miller, The Seventy-six Standard Solutions with Examples, The TRIZ journal, February, 2000, http://www.triz-journal.com.

2. Altshuller G., The Innovation Algorithm, TRIZ, systematic innovation and technical vreativity, Technical Innovation Center, INC, Worcester, 1999

3. Kalevi Rantanen, Levels of solutions, The TRIZ Journal, Dec. 1997, http://www.TRIZJournal.com

4. Savransky S D, Engineering of Creativity, New York: CRC Press, 2000

5. Tan Runhua, Progress of some problems in product design for innovation, Chinese Journal of Mechanical Engineering, Sep., 2003

6. Computer Aided Inventive Problem Solving System, InventionTool1.0, The number of computer software register: 2003SR2694

7. Hassan Gomaa, An Object-Oriented domain ananlysis and modeling method for software reuse, 0073-1129-1/92, 1992 IEEE. 